\title{
Salud en Sonora desde una perspectiva de género: retos y propuestas
}

\author{
Catalina A. Denman* \\ Ma. del Carmen Castro V. ${ }^{*}$ \\ Patricia Aranda*
}

Resumen: El presente artículo es una reflexión en torno a las desigualdades de género que repercuten en la salud de los sonorenses. Se partió del análisis de las cinco causas principales de muerte para dos grupos de edad: 15 a 24 y 25 a 34 años. Tal delimitación se hizo con el afán de acotar y esclarecer las diferencias en dicho grupo de edad, de ninguna manera se pretende afirmar que el mismo estudio no resulta pertinente para el resto de la población. El análisis de las diferencias entre hombres y mujeres, desde una perspectiva de género, aporta una mejor comprensión de los problemas de salud y sus condicionantes socioculturales; para ambos se definen roles, responsabilidades y obligaciones diferentes, se establecen normas sociales que determinan su acceso desigual al poder social, económico y político. Se incluye una descripción conceptual breve, datos estadísticos y su análisis, así como un apartado final sobre retos y propuestas.

Palabras clave: género, inequidad, salud, mortalidad, salud reproductiva, violencia.

Abstract: This article offers a reflection on gender inequalities that affect the health of Sonora's population. We analyzed the five leading causes of death for two age groups: 15 to 24, and 25 to 34. This design was used for the purpose of defining and clarifying the differences within this age group, though we do not affirm in any way that the same analysis is irrelevant to the rest of the population. An analysis of the differences between men and women, from a gender perspective, contributes to a better understanding of health issues and their socio-cultural cons-

\footnotetext{
* Profesoras-investigadoras del Programa Salud y Sociedad de El Colegio de Sonora. Agradecemos el apoyo de Gerardo Álvarez, Elsa Cornejo y Myriam García para la elaboración de este texto, así como de los evaluadores anónimos. Correspondencia: Obregón 54, colonia Centro, C. P. 83000, Hermosillo, Sonora, México. Teléfono 01(662) 259-53-00, extensiones 2252, 2222 y 2224, respectivamente. Correos electrónicos: cdenman@colson.edu.mx; ccastro@colson.edu.mx; pag@colson.edu.mx
} 
traints; different roles, responsibilities and obligations are established for men and women, in addition to social norms that determine an unequal access to social, economic and political power. We include a brief conceptual description, the analysis of statistical data, as well as a final section on challenges and proposals.

Key words: gender, inequality, health, mortality, reproductive health, violence.

\section{Introducción}

Varios ejes de desigualdad atraviesan las formas de enfermar y de atender la salud en Sonora; entre los más importantes están los económicos, étnicos y de género. Es crucial reflexionar sobre ellos, pues entender y atender las desigualdades mejoraría las condiciones de salud para la población, y las aportaciones al conocimiento serían benéficas. La finalidad de este artículo es reflexionar sobre las desigualdades de género, que repercuten en la salud de los sonorenses. Se partió del análisis de las principales causas de muerte para dos grupos de edad: 15 a 24 y 25 a 34 años. El objetivo de tal delimitación es acotar y esclarecer las diferencias en dichos grupos de edad, de ninguna manera se pretende afirmar que el mismo estudio no resulta pertinente para el resto de la población. Se seleccionó el grupo de edad de 15 a 34 años en particular, porque en él ocurre la mayor parte de los eventos reproductivos y se identifica una serie de prácticas de riesgo, que desembocan en muertes por violencia y viH-sida, que lo afectan predominantemente.

Se hará referencia a causas de mortalidad particulares incluidas en la clasificación general, debido a su importancia en la desigualdad social y de género, como son los cánceres femeninos, la muerte materna y la violencia doméstica, así como los accidentes y su relación con el alcohol, agresiones y suicidios.

El género es una construcción social, ${ }^{1}$ que define de manera diferente roles, responsabilidades y obligaciones para hombres y mujeres, establece normas que determinan su acceso desigual al poder social, económico y político (Lamas 1996). Entre otras cosas, los estudios de género han contribuido a mostrar cómo la salud y el bienestar de ambos son moldeados por las construcciones sociales, que definen las formas de ser mujer y de ser hombre (Szasz 2003; ops 2004). Dicho análisis rebasa la visión biologicista, incluso la sociobiologicista, que plantea que fenómenos como la violencia se deben a la naturaleza humana, por ejemplo, al tipo de hormonas que produce cada sexo (De Souza Minayo y Ramos de Souza 1999).

A diferencia de otros textos sobre género y salud centrados en el tema de las mujeres, en este artículo también se busca llamar la atención sobre las causas de mortalidad que afectan a los hombres, asociadas con la inequidad de género.

\footnotetext{
${ }^{1}$ Los textos clásicos para esta discusión pueden consultarse en Lamas (1996).
} 
Una de las consecuencias de la creciente globalización es la incorporación de los acuerdos internacionales, en temas de relevancia social, al marco legal mexicano. Las conferencias internacionales de la mujer celebradas en México en 1975, en Nairobi en 1985, en Copenhague en 1990 y en Beijing en 1995, y la Conferencia de Población y Desarrollo en El Cairo, en 1994, produjeron importantes plataformas de acción, refrendadas por México, con la intención de mejorar las condiciones de las mujeres. Integran, junto con la modificación al artículo $4^{\circ}$ constitucional en 1984, el marco de referencia para la elaboración de leyes, normas y programas dirigidos a construir la equidad de género en México. Una década después se crearían los institutos de la mujer estatales y el nacional, ${ }^{2}$ las coordinaciones de equidad y género en la Secretaría de Salud, entre otros instrumentos, para lograr los objetivos planteados en los acuerdos internacionales, asumidos por México, y realizados muchas veces en estrecha colaboración con instituciones académicas, grupos de profesionistas, así como numerosas organizaciones de la sociedad civil, cuyas luchas dentro del movimiento amplio de mujeres contribuyeron a sensibilizar y movilizar a gobierno y sociedad.

En septiembre de 2000, en el seno de la Organización de las Naciones Unidas (ONU), se establecieron las Metas del Milenio para alcanzarse en 2015, las cuales incluyen la promoción de la igualdad de género y la autonomía de las mujeres (PNUD 2004; Grown, Gupta y Kes 2005). Con estas referencias se ilustra la institucionalización del concepto de género en políticas públicas que incidan en la normatividad, la legislación y los derechos en México. Al menos en términos programáticos y normativos, se cuenta con el soporte de un marco legislativo y lineamientos básicos para la inclusión de una perspectiva de género más equitativa. Sin embargo, el proceso cotidiano de construcción de relaciones de género nuevas, como todo proceso cultural, es más largo y complejo.

\section{Inequidad de género y salud}

La perspectiva de género permite analizar el estado de la salud de hombres y mujeres y sus posibles determinantes; identificar los obstáculos de acceso a los servicios y los recursos de atención destinados a ellos; evaluar el efecto de las políticas y programas vigentes en la salud; conocer la organización de la fuerza de trabajo en los servicios de salud y sus salarios y la participación política de ambos (Gómez Gómez 2002). También, permite esclarecer los patrones culturales por los que se rigen hombres y mujeres de los distintos grupos de edad. Hay que enfatizar el carácter relacional de la perspectiva de género entre las distintas causas de vivir, enfermar y morir de hombres y mujeres.

En el presente texto, se asume la definición de la Organización Mundial de la Salud (OMS) sobre la inequidad: "Las desigualdades que son innecesarias, evitables

\footnotetext{
2 El Instituto Nacional de las Mujeres se creó en 2001, y en 1998 el Instituto Sonorense de la Mujer.
} 
e injustas" (Whitehead 1990, 5). Entonces, la equidad de género no se refiere a que mujeres y hombres deban enfermar y morir por las mismas causas y en tasas iguales, significa la "ausencia de diferencias evitables en las oportunidades de sobrevivir y disfrutar de salud, y en la probabilidad de no padecer enfermedad, discapacidad, ni muerte prematura por causas prevenibles" (ops 2004, 15). En complemento, la equidad de género en salud estaría dada por la asignación de recursos con base en la necesidad diferenciada de ambos sexos, para recibir servicios de salud según la etapa de vida y condición socioeconómica, sin que dependa de la capacidad de pago para obtenerlos (ops 2004, 17).

Gómez Gómez (2002) señala que los principales problemas y desigualdades de género, enfatizan que las mujeres están más expuestas, por su función productiva y reproductiva en la sociedad, a más riesgos que los hombres, y sus oportunidades para disfrutar de un estado de salud adecuado son menores. Esto se observa, por ejemplo, en las muertes prevenibles por cáncer de mama y cervical y debido a causas relacionadas con la reproducción y el incremento de las tasas de mortalidad por VIH-sida. Las condiciones precarias de vida incrementan las dificultades para acceder oportunamente a los servicios de salud, además del costo diferenciado ocasionado por tales riesgos.

Además, en las investigaciones respectivas se subraya que las mujeres asumen mayor responsabilidad en el cuidado de la salud de la familia, y que su trabajo no es remunerado económicamente; ellas cuentan con menos recursos para pagar los servicios de salud y sus condiciones son más difíciles para la toma de decisiones.

Por su parte, los hombres enfrentan una serie de riesgos de salud al sujetarse a los modelos de masculinidad dominantes, y que se manifiestan en tasas de muerte más altas por causas violentas, como homicidios, suicidios, accidentes y prácticas en la sexualidad, que los expone a una mayor probabilidad de contagio de infecciones de trasmisión sexual, como el viH-sida.

En el apartado siguiente se analizarán algunas de las principales causas de muerte de los sonorenses, y sus posibles condicionantes socioculturales y económicas. Esto hace que mujeres y hombres enfrenten riesgos diferenciados, según construcción de género. Para contextualizar dicha información, se hará referencia a tasas similares en los estados fronterizos, así como a las de todo el país y ocasionalmente a las del vecino estado de Arizona.

\section{Mortalidad en Sonora: un análisis de género}

En las últimas tres décadas, el perfil de morbilidad y mortalidad en Sonora muestra cambios sustanciales que es preciso señalar. Ya en un análisis sobre salud y enfermedad en la década de los ochenta (Denman y Haro 1990, 275), se concluía que el perfil epidemiológico del estado reflejaba "una serie de tendencias encontradas compatibles con lo que ha sido llamado un esquema de "modernización defectuosa”". Esto es, la presencia de enfermedades crónico-degenerativas en convivencia con las infecto-contagiosas, como las principales causas de morbilidad y 
mortalidad. Entre las tendencias señaladas por los autores para este milenio están las enfermedades cardiovasculares, diabetes, VIH-sida, cáncer, los accidentes, la violencia y las toxicomanías, observadas actualmente en las estadísticas entre las principales causas de muerte.

Uno de los cambios más sobresalientes es la disminución de la natalidad. En 1970, la tasa bruta en Sonora fue de 45.7 y 27.0 en 1995 (Castro Luque 2000); para 2006, el Consejo Nacional de Población (CONAPO 2005) la estimó en 17.8. Algunas de las condiciones socioculturales que han permitido dichos cambios son mayor información sobre la sexualidad, más acceso y aceptación del uso de los anticonceptivos, en un contexto de más escolaridad de la población y cambios en el desarrollo económico del país. Las prácticas de anticoncepción han variado en las últimas décadas entre hombres y mujeres; sin embargo, las mujeres se han encargado de esta responsabilidad, debido a que son ellas quienes por su función reproductiva se han mostrado más motivadas en cumplirla. Entre los métodos definitivos de anticoncepción, la salpingoclasia en México y Sonora se ha ido posicionando en primer lugar entre las mujeres de 15 a 49 años; a escala nacional, en 2002, por cada 19 cirugías de éstas, sólo se realizaba una vasectomía. ${ }^{3}$

Sobre esta transición reflejada en la demografía, pero también en el ámbito familiar e individual, no ha habido, en Sonora, una reflexión sistemática, quizá debido al crecimiento de la población, a pesar de la disminución de la natalidad. Hay que pensar en los cambios radicales que esta baja en la natalidad y sus efectos concomitantes produjeron en familias, escuelas, roles de género, participación en el trabajo y la política de mujeres, y también en los asuntos pendientes que gobierno y sociedad tendrán que atender para enfrentar estas transformaciones.

La mortalidad materna es un indicador fundamental de desarrollo social y sanitario, y es el que explica por excelencia las desigualdades sociales, incluida la inequidad de género; asimismo, es un problema reconocido mundialmente y sobre el que se toman acuerdos para dar importancia especial a la calidad de la salud de una población, por tanto los organismos internacionales y los países signatarios de acuerdos lo han asumido como un compromiso central de trabajo. En Sonora, la mortalidad materna no es considerada un problema de salud grave, sin embargo, según estadísticas del último quinquenio, ha habido un estancamiento relativo en dicha tasa (véase gráfica 1), lo que refleja una ineficiencia persistente en las medidas preventivas y de educación en la salud de las mujeres, y falta de calidad en la atención, lo que se traduce en un seguimiento inadecuado de los casos (Cerón 2005).

El Informe Salud: México 2001-2005 (Secretaría de Salud 2006), señala que la mortalidad materna en México disminuyó 10.5 por ciento entre 2001 y 2005, indicador que pronostica el probable incumplimiento de una de las metas nacionales más

\footnotetext{
3 Lourdes Quintanilla de la Dirección General de Salud Reproductiva de la Secretaría de Salud (ss), informó que en México, en 2002, se realizaron 1131000 procedimientos definitivos, de los cuales 5426 fueron vasectomías, lo que se traduce en una intervención en varones por cada 19 en mujeres (Magalli 2003). Otros datos señalan que de 1976 a 1995, la vasectomía pasó de 0.8 a 1 por ciento, y se incrementó a 1.9 para 2004 .
} 
importantes, que se establecieron en los Objetivos de Desarrollo del Milenio (ODM), es decir, la reducción de la razón de mortalidad materna en tres cuartas partes, entre 1990 y 2015. En México, esto significa reducir las muertes maternas de 89 a 22.3 por 100 mil nacimientos. Si bien Sonora comparte la tendencia discreta a la disminución nacional en el mismo periodo, no ha logrado alcanzar tasas más aceptables. De acuerdo con cifras del Sistema Epidemiológico y Estadístico de Defunciones de la Secretaría de Salud Pública de Sonora, en 2000 se reportó en el estado una razón de mortalidad materna de 28.8 por cada 100 mil nacidos vivos registrados (NVR), y en 2004 subió a 31.7. Como se puede ver en la gráfica 1, entre 2000 y 2004, la razón de mortalidad materna de Sonora fue menor que la nacional, con un promedio de 33.4, casi 14 puntos por debajo del promedio nacional, que fue de 47.24 por cada 100 mil nacidos vivos registrados. Se aprecia una alza en los primeros dos años, y una disminución hasta 31.7 en 2004, estos datos representan el tamaño del reto que implica para el estado construir condiciones para que las mujeres no mueran durante el embarazo y el parto, tarea que no debe ser tan compleja cuando se cuenta con una cobertura alta de servicios básicos.

\section{Gráfica 1}

Mortalidad materna en México y Sonora, 2000-2004

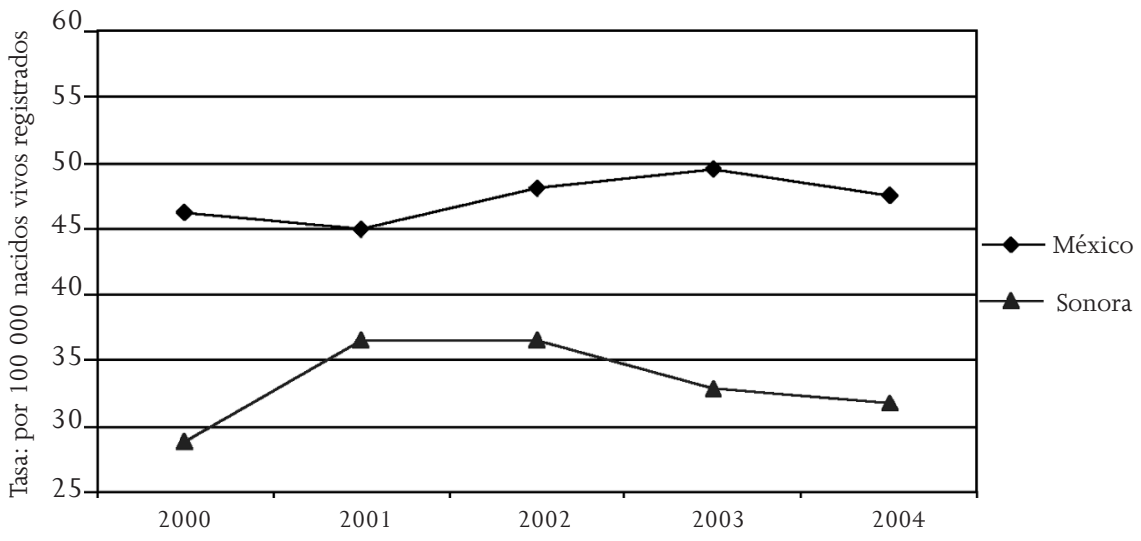

Para México:

Fuente numerador: Sistema Nacional de Información en Salud. Cubos dinámicos de mortalidad. Secretaría de Salud.

Fuentes denominador: Dirección General de Estadística: Dirección General Adjunta de Estadística Sociodemográficas; Dirección de Análisis y Estudios Demográficos; Dirección Regional Noroeste; Dirección de Estadística de Control y Desarrollo Estadístico. INEGI.

Para Sonora:

Sistema Epidemiológico y Estadístico de Defunciones. Bases de datos 2000-2005. Secretaría de Salud Pública. 
Por ejemplo, al comparar la mortalidad materna en la región fronteriza mexicana del norte, en 1999 la tasa fue entre 30 y 40 muertes por 100 mil NVR, mientras que en Estados Unidos fue de 10 (Comisión de Salud Fronteriza México-Estados Unidos 2003), es decir, tres y cuatro veces menor que en la población mexicana.

A partir de un análisis desde la perspectiva de género, es posible reconocer algunas de las condicionantes adversas que señalan los estudios epidemiológicos y socioantropológicos sobre prácticas cotidianas y culturales por las que muchas mujeres fallecen debido a complicaciones durante el embarazo, parto o puerperio, la mayor parte de ellas prevenibles y por lo tanto evitables.

De acuerdo con información del Fondo de Población de las Naciones Unidas (UNFPA, por sus siglas en inglés) de 2004, las adolescentes entre 15 y 19 años de edad corren el doble riesgo de fallecer debido a complicaciones del embarazo, parto y puerperio que las de 20 y 30; las menores de 15 son quienes presentan cinco veces más riesgo. Se considera que 15 por ciento de los embarazos puede presentar complicaciones, pero el riesgo de que desemboquen en muerte se concentra principalmente en la población menor de 15 años de edad (UNFPA 2004, 76).

Se puede representar a las mujeres que mueren por falta de atención oportuna debido a complicaciones durante el proceso reproductivo, así: las que vivían en la pobreza y le dieron prioridad a la atención del resto de su familia; las que no contaban con información médica clara y actualizada sobre riesgos principales y síntomas que cuidar y aquéllas a quienes sus condiciones laborales, familiares y culturales, a la par de la saturación de los servicios médicos a los que pudieron acudir — si tuvieron acceso-, no les facilitaron una atención prenatal de calidad para identificar y atender oportunamente los problemas. Muchas de ellas vivían en zonas alejadas de los servicios médicos, pertenecían a diferentes grupos étnicos y eran menores de 15 años. A la par, su autoestima frente al resto de su familia y grupo social fue mucho menor, por estar establecido que lo masculino es de mayor valor, lo que reforzó, a partir de comentarios de las personas que conformaban sus redes sociales, que los posibles síntomas de alerta fueran tomados como naturales del embarazo, y la resignación de la futura madre a padecerlos evita que sean identificados como problemas posibles. En resumen, son mujeres cuyas dificultades de vida les impide obtener información oportuna sobre los riesgos, síntomas y medidas de prevención para dichas complicaciones, y por ende una exigencia menor en la atención de calidad en los servicios de salud.

Los adolescentes viven una etapa que los define y ubica frente a sus pares, padres y el resto de la sociedad, y evidencian de manera nítida su pertenencia a los patrones socioculturales que se espera exprese cada sexo, según la construcción de su identidad de género; de igual forma, durante esta edad muchos sufren conflictos familiares y sociales, generadores de angustia, ansiedad y depresión, y no siempre son resueltos de la mejor manera.

El análisis de las estadísticas muestra que entre las primeras cinco causas de mortalidad de la población sonorense, del grupo de edad de 15 a 34 años, en primer lugar están las muertes violentas (accidentes, homicidios y suicidios), sobre todo en la población masculina, y en el caso de las mujeres se suman las relaciona- 
das con el proceso reproductivo. Al hacer la acotación por grupo de edad de 15 a 24 años y de 25 a 34, las características son más nítidas para comprender las diferencias de género en la conformación social de las prácticas y riesgos para la salud.

Según se observa en el cuadro 1, las tres primeras causas de muerte de los varones son accidentes, agresiones y suicidios, que no son biológicas, sino están íntimamente relacionadas con el entorno urbano y violento en el que transcurre la cotidianidad de la mayor parte de la población en la entidad. Para este mismo grupo, las mujeres corren un riesgo importante de morir por causas relacionadas con el embarazo, parto y puerperio, que se ubican en el cuarto lugar antecedidas por los accidentes y suicidios. Los datos de muerte, relacionados con el proceso reproductivo, incluyen parte de la mortalidad clasificada como muerte materna. ${ }^{4}$ Cabe mencionar que según estadísticas nacionales, en Sonora, más de 30 por ciento de los partos atendidos son de mujeres de 19 años o menos, ocupa así el cuarto lugar de la lista, después de Baja California, Chihuahua y Coahuila (Secretaría de Salud 2006).

\section{Cuadro 1}

Primeras cinco causas de mortalidad en el grupo de 15 a 24 años de edad, por sexo, en Sonora, 2003

\begin{tabular}{|c|l|l|}
\hline Causas & Mujeres & Hombres \\
\hline 1 & Accidentes & Accidentes \\
2 & Lesiones autoinfligidas intencionalmente & Agresiones \\
3 & Tumores malignos & Lesiones autoinfligidas intencionalmente \\
4 & Embarazo, parto y puerperio & Tumores malignos \\
5 & Agresiones & Enfermedades del corazón \\
\hline
\end{tabular}

Fuente: INEGI (2005).

Los accidentes, entre los cuales los de tráfico de vehículo de motor resultaron los más frecuentes, fue la primera causa de muerte entre los jóvenes de 15 a 24 años de edad en ambos sexos en Sonora, durante 2003. El consumo de alcohol se asocia a este tipo de "accidentes", que en gran medida son sucesos prevenibles. De acuerdo con los datos del Informe mundial sobre prevención de los traumatismos causados por el tránsito: resumen (OMs 2004), la frecuencia de mortalidad por esta causa es bastante mayor en hombres que en mujeres. Hay mucho por investigar sobre los accidentes en Sonora, y las características de las personas que sufren sus consecuencias. La

\footnotetext{
${ }^{4}$ Se define muerte materna como "la muerte de una mujer mientras está embarazada o dentro de los 42 días después de la terminación del embarazo, independientemente de la duración y lugar del mismo, producida por cualquier causa relacionada o agravada por el embarazo o su manejo, pero no por causas accidentales o incidentales" (OMS 1992, citada por Elu y Santos 2004, 45).
} 
entidad se ubicó entre los primeros diez estados con más muertes por esta causa, la tasa estandarizada de mortalidad de los hombres sonorenses fue de 30.7 por 100 mil habitantes en 2004, y en el mismo año, la de las mujeres fue de 8.2 (Secretaría de Salud 2006, 174). No obstante, el mismo informe señala que en el país, de 2001 a 2005, el aumento de las muertes de hombres por esta razón fue de 1 por ciento, y de 6 en las mujeres. Sonora podría seguir un patrón similar, habría que investigar si el aumento de actividades de las mujeres fuera del espacio doméstico se relaciona con más riesgo de fallecer en accidentes de tráfico.

También, es de consideración la cantidad de muertes debido a "lesiones autoinfligidas intencionalmente", es decir, los suicidios, que en las mujeres ocupa el segundo lugar en decesos, y en los hombres el tercero. Aunque la estadística de la Secretaría de Salud para los estados abarca a la población de todas las edades, es sorprendente que Sonora ocupe uno de los primeros lugares de mortalidad según tasa de suicidios ocurridos; en los hombres es de 11.3 por 100 mil habitantes, y en las mujeres de 0.9. Estas diferencias, ambas reflejadas como de las primeras causas, deben entenderse según construcción genérica entre hombres y mujeres. En los primeros, la cultura violenta se expresa en tasas más altas de suicidio, aunque entre las mujeres esta causa de muerte ha ido aumentando paulatinamente, según da cuenta la Secretaría de Salud $(2006,136)$; el alza ha sido de 95 por ciento en comparación con 67 en hombres de 1990 a $2004 .^{5}$

Dichas diferencias también expresan la gravedad de los conflictos existenciales en una etapa formativa de la vida; en el caso de los varones, la falta de comunicación de las emociones puede resultar más grave. De Keijzer (2003) señala que dicha incapacidad de expresión de los sentimientos, miedos y conflictos interiores, que podría considerarse una expresión femenina es característica del modelo hegemónico de masculinidad, que según la definen los estudiosos, es "un conjunto de atributos, valores, funciones y conductas que se suponen esenciales al varón en una cultura determinada" (De Keijzer 2003, 2), entendida como un proceso de socialización que les incita a una vida de mayor independencia, agresividad, competencia e incorporación de conductas violentas y temerarias en aspectos tan diversos como la relación con vehículos, las adicciones, las relaciones familiares y la sexualidad. 6

El tema del embarazo adolescente es una preocupación constante para la sociedad y sus servidores públicos, sin embargo, es preciso reconocer la dimensión social que se entreteje en torno a esta temática. Al hacer un recuento de las prácticas reproductivas en la entidad, se observa un descenso importante en el número de nacimientos, lo cual incluye una disminución en el embarazo a edades tempra-

\footnotetext{
${ }^{5}$ En un estudio en Sonora de 1990 a 1997, las mujeres intentaron con más frecuencia el suicidio, pero fue mayor cantidad de varones quienes alcanzaron su objetivo (Mayoral 2004, 757).

${ }^{6} \mathrm{Y}$ aun cuando los estudios se refieren a las masculinidades en plural, para dar cuenta de las diversidades a partir de diferencias de nacionalidad, clase, edad, etnia, migración, orientación sexual, etcétera, de acuerdo con el mismo autor, "cada hombre se encuentra enclavado en un continuum en donde comparte, en grados distintos, lo común y lo diverso con otros hombres" (De Keijzer 2003, 3).
} 
nas. Si se analiza a largo plazo, se ve que en lugar de encontrar un aumento proporcional al tamaño de la población en edad reproductiva que se embaraza antes de los 19 años, hay un descenso general con matices por grupos socioeconómicos y niveles de educación, y ciertamente con una disminución de menor proporción en las mujeres adolescentes (menores de 19 años), respecto a otros grupos de edad. La anticoncepción es más frecuente una vez que se ha decidido que se tiene el número de hijos deseado, y no tanto para alargar el lapso entre gestaciones. La construcción social de género de las mujeres en torno a la maternidad abona, en gran medida, la consolidación de prácticas en este sentido. Lo que ha cambiado drásticamente son los significados y las valoraciones del embarazo en este grupo de edad, así como las condiciones sociales a las que se enfrentan quienes inician su vida reproductiva de manera temprana.

Respecto al comportamiento de las causas de muerte en el grupo de edad de 25 a 34 años, llama la atención que la violencia sea el principal ingrediente para la población masculina, según se aprecia en el cuadro 2. El caso de agresiones para ambos grupos de edad, las muertes de hombres clasificadas como producto de agresiones, se mantiene en segundo lugar. Para el caso de las mujeres, en el grupo más joven, representa la quinta causa de muerte, pero desaparece entre las primeras cinco en el grupo de 25 a 34 años. Habría que preguntarse cuántos casos de mujeres muertas, en el grupo más joven, no están relacionadas con conductas de discriminación y homicidios por violencia doméstica. Esto se retomará un poco más adelante.

En el grupo de mujeres de 25 a 34 años, las muertes por accidentes se mantienen como la única causa relacionada con decesos violentos; las cuatro restantes son enfermedades crónico-degenerativas, siendo los tumores malignos las que ocupan el primer lugar. ¿A qué se deben estas grandes diferencias? De Keijzer explica que en su formación, los hombres asumen prácticas de riesgo que los ponen en desventaja, e incrementan sus probabilidades de morir prematuramente por causas íntimamente relacionadas con su identidad de género, con un gran contenido de prácticas violentas. ${ }^{7}$ Los jóvenes en este grupo de edad deben demostrar su pertenencia de género con el consumo de alcohol o drogas, con un mayor arrojo en sus prácticas de manejo, y con el enfrentamiento de la agresión de sus pares con más facilidad.

Aunado al panorama anterior, uno de los hechos más dramáticos de los últimos diez años es la violencia de género. Su expresión más cruda son las muertes de mujeres y hombres, que construyen los hechos de feminicidio y homofobia en esta entidad fronteriza. El primero suele concentrarse en los casos de violencia de hombres hacia sus parejas. Una explicación parcial se basa en los roles de género cambiantes, expresados en una incorporación mayor de las mujeres al mercado laboral, más desempleo entre la población masculina, aunado al reconocimiento de la violencia como problema social y de salud, y su consecuente visibilización.

7 Un estudio ilustrativo realizado en Sonora, sobre masculinidad y prácticas de riesgo en el trabajo, es el de Calvario (2003). 
A pesar del reconocimiento del problema de la violencia de género como tal, actualmente apenas se estudia, y las personas más vulnerables se enfrentan a la ausencia de un seguimiento sistemático y consistente de cualquiera de sus manifestaciones, por las instancias correspondientes (World Health Organization 1997). En 2004 se registraron en Sonora 22 homicidios dolosos de mujeres, reportados por las procuradurías generales de justicia de la entidad; en Sonora, las tasas de homicidios de mujeres en el grupo de edad de 0 a 14 años están entre las más elevadas del país (H. Congreso de la Unión, Cámara de Diputados, LIX Legislatura 2006, 308).

\section{Cuadro 2}

Primeras cinco causas de mortalidad en el grupo de 25 a 34 años de edad, por sexo, en Sonora, 2003

\begin{tabular}{|c|l|l|}
\hline Causas & Mujeres & Hombres \\
\hline 1 & Tumores malignos & Accidentes \\
2 & Accidentes & Agresiones \\
3 & Enfermedades del corazón & Lesiones autoinfligidas intencionalmente \\
4 & Enfermedades cerebrovasculares & Enfermedad por viH \\
5 & Diabetes mellitus & Enfermedades del corazón \\
\hline
\end{tabular}

Fuente: INEGI (2005).

La violencia tiene repercusiones directas en la salud y relaciones cotidianas de quienes viven en pareja. La Encuesta Nacional de la Dinámica de las Relaciones en los Hogares 2003 (ENDIREH) ${ }^{8}$ realizada por el INEGI y el Instituto Nacional de las Mujeres (2004), revela que en Sonora (véase cuadro 3) casi la mitad de las mujeres mayores de 15 años (49.8 por ciento), que viven con su pareja en el hogar, sufren violencia de algún tipo por parte de su compañero o esposo. De ellas, 8.4 por ciento son objeto de agresiones físicas y 7.1 de agresiones sexuales.

Aun cuando los datos de la encuesta se presentan con advertencias sobre la falta de información en algunos de ellos, permite contar con un primer indicador del comportamiento del fenómeno de la violencia contra las mujeres que viven en pareja en la entidad. Es necesario considerar que las relaciones violentas expresadas son solamente la punta del iceberg en torno a esta problemática, ya que la misma dependencia económica y emocional que implica la relación de pareja dificulta las respuestas generalizadas y más aún los registros de las demandas legales interpuestas. Las limitaciones para el registro de esta información son mayores en los entor-

8 Dicha encuesta es representativa para 11 entidades federativas, entre ellas Sonora. 
nos rurales, por lo que es necesario tomar con precaución la información. Con todo ello, el reporte de que al menos la mitad de las mujeres declararon sufrir algún tipo de violencia alerta sobre la magnitud de un tema, que hasta fechas recientes se mantenía en el ámbito privado.

\section{Cuadro 3}

Proporción de mujeres con al menos un incidente de violencia en los últimos 12 meses, por tipo de violencia y localidad en Sonora

\begin{tabular}{|l|r|r|r|}
\hline & & $\begin{array}{c}\text { Nacional } \\
\%\end{array}$ & $\begin{array}{c}\text { Sonora } \\
\%\end{array}$ \\
\hline \multirow{2}{*}{ Mujeres con algún tipo de violencia } & Total & 46.6 & 49.8 \\
& Urbana & 48.1 & 51.0 \\
& Rural & 41.0 & 43.1 \\
\hline Emocional & Total & 38.4 & 41.3 \\
& Urbana & 39.4 & 42.7 \\
& Rural & 34.6 & 33.9 \\
\hline \multirow{2}{*}{ Económica } & Total & 29.3 & 31.8 \\
& Urbana & 30.9 & 32.4 \\
& Rural & 23.6 & 28.7 \\
\hline \multirow{2}{*}{ Física } & Total & 9.3 & 8.4 \\
& Urbana & 9.3 & 8.1 \\
& Rural & 9.5 & 9.7 \\
\hline \multirow{2}{*}{ Sexual } & Total & 7.8 & 7.1 \\
& Urbana & 7.5 & 7.1 \\
& Rural & 9.0 & 6.8 \\
\hline
\end{tabular}

Fuente: INEGI e Instituto Nacional de las Mujeres (2004).

* Se refiere a las mujeres de 15 años y más con pareja residente en el hogar.

Otro problema de salud, asociado a las diferentes formas en las que hombres y mujeres viven y practican su sexualidad, se refiere a las infecciones de trasmisión sexual y el viH-sida; colocados en la cuarta posición como causa de muerte en la población masculina, y se vincula a las prácticas sexuales poco seguras que ponen en riesgo y aumentan la probabilidad de contagio a su contraparte femenina. Vale subrayar que en las relaciones de pareja, específicamente en las sexuales, se establecen también las relaciones de poder, expresadas en ocasiones en sexo no consensuado o forzado, o bien limitan la decisión de la compañera en su insistencia en el uso del preservativo, ya que este tipo de solicitud se interpreta como una 
expresión de desconfianza y sus posibles prácticas riesgosas con otras parejas; se considera también que el uso de condón se relaciona con la promiscuidad, prostitución y enfermedad.

La incidencia del viH-sida en los seis estados fronterizos de México con Estados Unidos en el año 2000 fue de 9.2 por 100 mil habitantes (Comisión de Salud Fronteriza México-Estados Unidos 2003). Para el mismo año, esta tasa fue de 3.1 por 100 mil en Estados Unidos. Sin embargo, el número de casos en México ha crecido rápidamente de 1980 a la fecha, y la tasa de mortalidad por esta causa en la frontera mexicana fue casi el doble de la nacional, que en 2000 fue de 4.2 por 100 mil habitantes, mientras que en la región fronteriza mexicana fue de 8.2.

En 2003 se reportó que en hombres entre los 25 y 34 años, el sida se encontraba en el cuarto lugar entre las causas de mortalidad en Sonora (véase cuadro 2), en comparación con otros estados fronterizos en los que se ubicaba en el tercero, como en Baja California, Nuevo León y Tamaulipas. El estereotipo del vIH-sida, como una enfermedad de hombres y homosexuales, se ha modificado poco a poco debido a los hechos reportados por el Consejo Nacional para la Prevención y Control del Sida (CONASIDA): 58.6 por ciento de quienes se contagiaron, lo hicieron a través de relaciones heterosexuales, 21.8 por contactos homosexuales y 17.4 por bisexuales, es decir, las prácticas sexuales de riesgo son ahora mayores en las relaciones heterosexuales, por consiguiente, las mujeres corren más riesgos, y ya no se trata de un problema de sexoservidoras, sino de mujeres y hombres en general (CONASIDA 2005).

Un último problema de salud que debe verse a través de la lupa de género son los decesos por cáncer en Sonora; éstos representan la quinta causa de muerte general en la población, con la consiguiente carga para los individuos, familias e instituciones de salud (Secretaría de Salud Pública de Sonora 2003). Pero en el estado es la primera causa de muerte de mujeres de más de 25 años. ${ }^{9} \mathrm{Al}$ respecto, podrían enumerarse una serie de condicionantes como la exposición a contaminantes, principalmente agroquímicos e industriales; una alimentación alta en grasas y proteínas y baja en fibras, vitaminas y minerales; así como más estrés y el incremento probable de prácticas sexuales sin protección, a la par de otras socioculturales, entre ellas las desigualdades sociales y de género.

Entre los tumores malignos, el cáncer mamario es la causa principal de muerte de mujeres mayores de 25 años, y el cáncer cervicouterino pasó del primer al segundo lugar desde 1998; la tasa se ha mantenido relativamente estable en los últimos cinco años. Ambas enfermedades han sido objeto de investigación en la región, y aunque se reconocen los múltiples factores biológicos presentes, también persisten elementos socioculturales que agravan la situación para las mujeres que enfrentan estos cánceres, que provocan muertes más dolorosas por ser evitables (Castro y Salazar 2001; Cartwright et al. 2004; Aranda 2005; Castro 2006). Se pueden señalar algunos elementos para subrayar cómo la focalización de las estrategias de intervención diseñadas desde una perspectiva de género pueden subsanar las

\footnotetext{
${ }^{9}$ Esta primera causa se mantiene hasta la edad de 65 años.
} 
deficiencias actuales de la atención en los servicios de salud, y contribuir en la disminución de la mortalidad.

Al igual que la muerte materna, las condiciones adversas por construcción de género dificultan aún más la atención oportuna a las mujeres. En los cánceres de mayor presencia, dichas condiciones incluyen también una menor apropiación del cuerpo y la sexualidad, que lleva al no reconocimiento de signos o síntomas de alerta. Para el cáncer de mama, esta dificultad se suma a las deficiencias de detección oportuna, y al seguimiento inadecuado de casos que presentan los servicios de salud, problema existente en Sonora así como en el resto del país. De acuerdo con los registros de la Secretaría de Salud, en el año 2000, en el país, ${ }^{10}$ sólo se diagnosticaron entre 5 y 10 por ciento de los casos de cáncer mamario en estadios tempranos (I), ${ }^{11}$ a diferencia de lo que sucede en los países desarrollados, donde alrededor de 50 por ciento de ellos se diagnostica en etapas tempranas, lo que ofrece mayores posibilidades de curación. En México, de 40 a 50 por ciento de los casos diagnosticados corresponden a etapas avanzadas (estadio III) o a un cáncer diseminado (estadio IV), que además de encarecer su atención, la probabilidad de sobrevivencia es muy baja, con el sufrimiento consecuente de las enfermas y una repercusión familiar y social mayor de las muertes.

Aquí surge una aparente contradicción de condicionantes, pues pese a que las mujeres comparten una cultura que por lo general no les permite conocer y tocar fácilmente su cuerpo, sobre todo las zonas genitales o erógenas, son ellas las primeras en detectarse alguna "bolita" en el pecho, y no el personal de los servicios de salud. No obstante, otros factores se conjugan en la demora de la búsqueda de atención. Algunos estudios reportan las dificultades de las mujeres en la búsqueda de atención como parte de su rol social, ya que la maternidad implica el cuidado de los miembros de la familia, pero no el cuidado de sí mismas (Szasz 1998). Lo anterior se acentúa cuando se combina con dificultades de acceso o de calidad de la atención en los servicios de salud. Esto explica en parte la situación de la detección tardía, a la que se le suman las condiciones de pobreza de muchos de los casos, lo que dificulta una atención oportuna y adecuada.

Szasz (2003) y Elu y Santos (2004) señalan que las mujeres viven su condición de subordinación tanto en el espacio doméstico como en los servicios de salud; en éstos, se presenta con una dimensión doble de mujeres y pacientes. Primero, por su condición de género que las hace sujetas de discriminación, luego al hecho de enfrentarse a una cultura jerárquica de los prestadores de servicios de salud, en la atención de su problema. Castro (2006) ha señalado que en Sonora dicha subordinación se vuelve más compleja entre la población carente de servicios de salud, pues se suma a su calidad de mujer y paciente, la de ser pobre para enfrentar la enfermedad.

\footnotetext{
10 Se incorpora esta referencia nacional, por carecer de estadísticas estatales. Ilustra mejor los argumentos, y se podría decir que dicho comportamiento es similar en el estado.

11 El cáncer de mama se clasifica en estadios I y II, donde es posible su resolución o una mayor sobrevida de las afectadas, y III y Iv que hacen más difícil su resolución y la sobrevida es menor.
} 
Las altas tasas de morbilidad y mortalidad por esta causa han rebasado a las instituciones de salud. Las deficiencias son importantes en medidas como la enseñanza de la autoexploración, el acceso a los servicios de detección oportuna, la práctica de la mamografía y un seguimiento efectivo de casos. Ello expresa la falta de recursos en el sector, aunado a la falta de orientación de medidas específicas que focalicen los esfuerzos posibles para esto, así como la necesidad de más educación sobre el cuidado de la salud. Frente a este tipo de problemas deben reconocerse las intrincadas redes de la desigualdad social, que partiendo de las diferencias de género y la condición socioeconómica, limitan un acceso oportuno a los servicios de salud.

Desgraciadamente, no existe el seguimiento pormenorizado del comportamiento de los factores que influyen en las tasas de mortalidad, igualmente válida para muchas causas de muerte como la materna, por tanto no es posible obtener un panorama más actualizado sobre este problema. Las estadísticas no alcanzan a "ir más allá” de sus causas inmediatas de mortalidad, según señalan Elu y Santos (2004).

El cáncer cervicouterino representa la segunda neoplasia que causa la muerte de mujeres mayores de 25 años en Sonora; la particularidad de este tipo de cáncer es que puede detectarse y tratarse a tiempo, y con mejor pronóstico, de manera que la mortalidad debido a él no está justificada en términos de la posibilidad diagnóstica y terapéutica.

De nuevo, la falta de información clara y oportuna de las mujeres sobre su cuerpo, así como las causas y el proceso de esta enfermedad, constituyen representaciones sociales acerca del Papanicolaou, y sobre cómo y cuándo buscar atención médica. Las investigaciones indican que hay deficiencias en la calidad de la atención en los servicios médicos, y que las mujeres prefieren tratarse con profesionistas femeninas, cuando el trato es cálido y les brinda confianza; también se ha demostrado que en estas situaciones, las mujeres solicitan mayor información al respecto (Castro 1998; Denman et al. 1999; Castro y Salazar 2001; Aranda 2005). En el caso de los hombres, habría que explorar la relación, desde una perspectiva de género, entre la detección y tratamiento oportuno del cáncer de próstata y la falta de una cultura integradora de los cuidados de la salud, como sucede para los cánceres femeninos.

A partir de que México ocupó uno de los primeros lugares de mortalidad en el mundo debido al cáncer cervicouterino en 1995, se dedicaron programas sociales para aumentar la cobertura y mejorar la calidad de la detección oportuna, que han resultado en menos muertes sobre todo en los últimos años. De 1995 a 2000 la disminución promedio fue de 1.8 por ciento; de 2000 a 2004 de 3.7 por año (Secretaría de Salud 2005, 14). A pesar de ello, durante 2003 continuaba siendo el cáncer más frecuente entre la población femenina en el país, y el segundo en Sonora.

Actualmente se reconoce que esta enfermedad es multifactorial, y en especial se asocia a infecciones de trasmisión sexual, como algunos tipos del virus del papiloma humano (VPH), que en 2003 tuvo una de las tasas de incidencia más frecuente en el ámbito nacional (6.8 por 100 mil habitantes). Los reportes epidemiológi- 
cos (Tirado-Gómez et al. 2005) señalan que el vpH está presente en 99.7 por ciento de los casos positivos de cáncer cervical, y en estudios nacionales se ha reportado que la presencia del vPH de alto riesgo ${ }^{12}$ aumenta 78 veces la probabilidad de presentarlo. Las prácticas sexuales sin protección y las infecciones de trasmisión sexual frecuentes se vinculan a su vez con prácticas culturales relacionadas con el cuerpo y la sexualidad.

Para el caso, podríamos ilustrar estas diferencias en la región, ya que en México, en 2000, se reportó una tasa de mortalidad de 22.4 por 100 mil mujeres en la franja fronteriza, mientras que un año antes, en la misma zona pero en Estados Unidos, la tasa fue de 2.9, de manera que el problema es mucho más severo del lado mexicano (Comisión de Salud Fronteriza México-Estados Unidos 2003, 21; Giuliano et al. 2001).

Para el estudio sociocultural del cáncer, tanto de mama como cervicouterino, los aportes de los abordajes de género al análisis de los hábitos de autocuidado, roles de género y las prácticas sexuales deben considerarse como parte nodal de la comprensión de estos padecimientos. Tal es el caso de las relaciones de poder establecidas entre las parejas que hacen de las prácticas sexuales una dimensión de riesgo para las infecciones de trasmisión sexual, donde las mujeres corren el mayor riesgo según su rol de género, en desventaja frente al varón. En otras palabras, las mujeres tienen menores posibilidades de protegerse que los hombres.

\section{Género y salud: retos y propuestas}

Para entender y atender estas muertes que pueden prevenirse o evitarse, hay algunas consideraciones sobre el tema de esta reflexión y que constituyen retos complejos para mejorar la salud en Sonora. Se acompañan de propuestas, necesariamente inconclusas, con referencia a la investigación y al quehacer político.

Las reflexiones siguientes deben enmarcarse en el reconocimiento de que la construcción de la salud no es un ámbito exclusivo de las instituciones de salud y seguridad social, que orientan la mayor parte de sus acciones y presupuesto a la atención curativa, sino que en ella influyen, en diferentes grados, tanto los individuos como las políticas generales de desarrollo.

La disminución de la mortalidad materna, de los decesos violentos en jóvenes, de los cánceres de mama y cervicales repercutiría positivamente en el resto de la población, no sólo en las mismas familias, sino en las instituciones de salud, en el descenso de los altos costos de atención implicados y en mayor seguridad para la población en general. El logro de estos objetivos es factible, si se comparan las tasas más aceptables que registran otros estados de la región, como se ha señalado en el texto. Para ello, también se requiere la consolidación de una infraestructura y prác-

\footnotetext{
12 Se entiende por VPH de alto riesgo oncológico o VPH-AR a los virus asociados con procesos agresivos y acelerados de transformación celular, vinculados principalmente a los serotipos 16, 18, 31, 33, 35, 52, 56 y 58 . Para más información véase Hernández Hernández et al. (2002).
} 
tica de investigación sobre salud en las propias instituciones del ramo y en el sector académico. Al respecto, la profundización tendría que considerar los debates contemporáneos, por ejemplo, sobre la multiculturalidad, lo público y privado, los nuevos referentes sobre las familias, la otredad o los movimientos ciudadanos, en un marco de rearticulación de las relaciones de poder en todas sus dimensiones.

La perspectiva de género es una categoría de análisis relacional, y de allí su importancia para reconocer las diferencias entre hombres y mujeres, pero sobre todo, para aclarar que éstas devienen en una desigualdad en la que las mujeres viven mayor subordinación frente a los varones. Esta situación afecta de manera diferencial su salud, y no opera de manera homogénea en todos los habitantes. Las comparaciones entre población rural y urbana reflejan esto nítidamente.

El estudio de los problemas principales de salud, que se reportan en las estadísticas de mortalidad, no puede desligarse de la pobreza, desempleo, acceso a los servicios médicos y concentración de la riqueza; en este artículo se ha señalado que las desigualdades de género sólo pueden comprenderse cabalmente en relación con las inequidades conjugadas en contextos que ponen en mayor o menor riesgo la salud y la vida de la población. El enfoque de género permite analizar los problemas complejos referidos, desde una perspectiva que toma en cuenta la existencia de procesos de socialización de hombres y mujeres, que fortalecen prácticas de mayor riesgo para unos y otras, así como las relaciones de poder que se construyen entre ambos.

De esta reflexión breve surgen las propuestas siguientes para investigación:

1. Los factores socioculturales relacionados con las muertes debido a accidentes y violencia, en poblaciones de mayor vulnerabilidad.

2. Los factores y condicionantes relacionados con la muerte materna en Sonora.

3. Las muertes por suicidio, desde una perspectiva de género.

4. Los significados y problemas sociales principales relacionados con el embarazo en adolescentes, dimensionando los datos epidemiológicos en el contexto de inequidades actuales y en los procesos de más largo plazo, al menos de dos generaciones.

5. La perspectiva de los actores relacionados con las políticas públicas sobre el VIHsida y sus propuestas.

6. Los obstáculos e instrumentos para implementar políticas públicas específicas, para la consideración de la perspectiva de género.

7. La implementación y supervisión de la capacitación del personal de salud sobre este abordaje.

8. Las acciones cotidianas de las instituciones educativas y en los medios masivos de comunicación, en temas relacionados con las causas de mortalidad principales, desde una perspectiva de género.

Se requieren, además, puentes que faciliten una socialización más efectiva de los resultados de investigación entre usuarios diferentes, como: gobiernos locales y estatales, el Poder Legislativo, organizaciones ciudadanas, el sector privado o los medios masivos de comunicación. 
Al trabajar en este artículo, y revisar informes y programas de gobierno, se constató la urgencia de considerar los factores sociales relacionados con las causas de mortalidad principales de la población entre 15 y 34 años de edad, pues en ellos se manifiesta que la perspectiva de género continúa ausente de manera efectiva y cotidiana, a pesar del compromiso asumido por el gobierno del país e indicado a las legislaturas estatales. La perspectiva de género se ha asumido en la normatividad federal, en el discurso, pero poco se ha traducido en acciones concretas y constantes de los gobiernos. En Sonora en particular, el tratamiento informativo y la planeación y programación de acciones también presenta estas limitaciones.

Uno de los mayores retos de los gobiernos actuales, tanto federal como estatales, es implementar la perspectiva de género de manera trasversal, no sólo desde las instituciones y los programas de salud, sino en todos los espacios que reproducen o fomentan los riesgos de muertes violentas y los problemas relacionados con la muerte materna en la población. El género como visión analítica y política no es una meta a alcanzar por única vez, sino un proceso permanente que aporta conocimiento sobre las desigualdades que viven hombres y mujeres en el país, para evitar tales condiciones.

Dicha trasversalidad debe basarse en información clara, oportuna y confiable, que implica rebasar la presentación de estadísticas desagregadas sólo por sexo. En vez de ello, sería de gran utilidad situarlas frente a otros condicionantes sociales que expliquen cuáles son las diferencias, por qué existen, cómo afectan a hombres y mujeres en condiciones diversas (por edad, etnia, trabajo, condición socioeconómica, tipo de trabajo, etcétera), y las leyes y programas vigentes y los que se necesita priorizar.

Se ha explicado que al entender mejor las causas y mecanismos que sostienen las diferencias entre los jóvenes para enfrentar prácticas de riesgo, será posible dirigir los programas e intervenciones con mayor efectividad. Las muertes violentas (accidentes, suicidios y agresiones), que se sostienen como las primeras causas de muerte entre los varones jóvenes en Sonora, indican que los programas de educación y atención a la salud deben focalizar más energía en concientizar acerca de los riesgos de estos patrones socioculturales, por un lado, y por otro, en planear estrategias educativas y de prevención con base en un acercamiento a quienes son sujetos de atención, para identificar con mayor certeza las conductas de riesgo de cada grupo, ya sea por sexo, edad, pertenencia étnica o tipo de trabajo desempeñado.

Abatir las muertes violentas entre la población joven, principalmente, debe ser un asunto central en la implementación de programas específicos dirigidos a ese objetivo. Además, el grupo de edad de 15 a 34 años se caracteriza por el dinamismo, fortaleza y productividad que representa para la sociedad en su conjunto. Mantener las tasas de mortalidad altas por causas prevenibles, y por ende modificables, refleja las disparidades en la construcción de género a las que se ve sometida la población masculina joven en el estado.

Entonces, si el reto es el abatimiento de las muertes violentas, se vuelve necesario adoptar estrategias, principalmente desde las instancias educativas y los medios masivos de comunicación, que influyan en las conductas de hombres y mujeres, 
que no sólo se han "naturalizado" considerándose normales, sino que se fomentan cotidianamente.

Otro reto por enfrentar, además de combatir la violencia, es la atención de los cánceres de mama y cervicouterino, que representan la primera causa de muerte en la población femenina mayor de 25 años. El argumento de que los recursos son limitados, esgrimido sistemáticamente para justificar la focalización de acciones en la curación más que en la prevención de las enfermedades, debe someterse a revisión y análisis serios que permitan redirigir algunos de esos recursos a la prevención, y partir del argumento, ampliamente aceptado por organismos como la OMS, de que ésta requiere de muchos menos recursos y su efecto positivo sería mayor a mediano y largo plazo, lo que abarataría la atención curativa, privilegiada actualmente. No está de más insistir en que para lograr un cambio positivo en el comportamiento de cualquiera de las tasas y las causas, las acciones deben acompañarse de una buena dosis de programas educativos.

En el caso de la mortalidad materna, en Sonora representa un reto disminuir la cantidad de mujeres que mueren debido a ella. Esta tasa se ha mantenido relativamente estancada en los últimos años, y sí se cuenta con infraestructura médica, que abarca a 98 por ciento de la población, y una cobertura hospitalaria elevada del parto, por lo que el índice de estas muertes no es aceptable. En el estado se han implementado algunas medidas que han tratado de mejorar la atención de las mujeres embarazadas, sin embargo, dichas intervenciones siguen siendo en la institución de salud, y no se han reflejado en una disminución de las muertes de mujeres por causas asociadas a la maternidad.

Para terminar, es necesario señalar un asunto más entre los muchos que se quedan en el tintero: la importancia de la capacitación de trabajadores de la salud en una perspectiva de género, que permita atender apropiadamente las necesidades diferentes de hombres y mujeres, y así mejorar su condición general de salud. La inmensa mayoría de los problemas atendidos en los servicios son diferentes para hombres y mujeres, como son distintos los contextos socioculturales que expresan los roles específicos de cada sexo, lo que afecta la vivencia de su enfermedad. Una mayor capacitación tendría que ir acompañada de más difusión de información sobre género y salud, que potenciara una mayor participación de las mujeres en la definición de prioridades de salud, la planeación de soluciones, programas y rendición de cuentas (OPS 2004). Según un estudio de Castro y Salazar (2000), en los años noventa la mitad de las acciones médicas estaban dirigidas a eventos reproductivos y a patología relacionada, por lo que, específicamente para este tema, se requiere fomentar mecanismos permanentes y efectivos de participación para las propias mujeres, más allá de los foros de consulta.

Finalmente, un asunto relativo a la investigación y la responsabilidad política de concretar los compromisos de equidad de género, es lo relacionado con la manera de organización de los sistemas de información nacional, estatal y local, y de ponerse al alcance de investigadores, políticos y sociedad civil. Ciertamente se reconocen avances en la última década, sin embargo, distintos sistemas de registro, nomenclatura, metodologías de cálculo y falta de capacitación de los encargados 
del llenado de formatos dificulta el acceso y la comprensión de los problemas de salud en el país, cuando se quiere analizar la desigualdad de género.

Las ideas previas pueden sintetizarse en torno al quehacer político y concretarse en las acciones prioritarias siguientes:

- Implementar urgentemente programas específicos dirigidos a evitar las muertes violentas de hombres y mujeres, y a disminuir la mortalidad materna.

- Implementar la perspectiva de género de manera trasversal, no sólo desde las instituciones y programas de salud, sino en los espacios donde se reproducen y fomentan riesgos para la población en general y la juventud en particular, para incentivar procesos culturales que fortalezcan las relaciones equitativas, solidarias y saludables.

- Dar prioridad al análisis de las desigualdades en la mortalidad, incluyendo la mirada que aporta la perspectiva de género en tanto es efectiva y pertinente para los problemas de salud; lo cual implica fortalecer los sistemas de información y análisis epidemiológico en las instituciones de salud, con recursos humanos altamente calificados.

- Priorizar, acorde con el marco normativo mexicano, el acceso a información y educación oportuna y científica en la prevención de prácticas sexuales de riesgo, que se beneficiaría de una coordinación efectiva entre instituciones de salud, de educación y organizaciones de la sociedad civil.

- Reconocer que el beneficio de la educación se refleja en una mejor salud para la población, con la inversión de más recursos en el sector educativo, así como el diseño de programas que aprovechen todos los espacios públicos para proyectos de educación en salud y de promoción de estrategias colectivas de prevención.

- Invertir mayores esfuerzos en la capacitación del personal de salud, y de los docentes en general, en torno a la importancia de considerar las diferencias de género en los servicios preventivos y de atención.

El reto para el problema de salud analizado en este artículo, asociado a las inequidades de género, puede expresarse en términos sencillos: disminuir las muertes maternas; las muertes por violencia, cánceres femeninos y VIH-sida en Sonora. Lo complejo es el cómo cerrar brechas entre ciudadanos e integrantes de la clase política para lograr un acuerdo que dé prioridad a la salud de la población; atender necesidades inmediatas a enfermedades sin desatender la salud pública; fomentar condiciones que promuevan una cultura no violenta, del cuerpo integral y de respeto a la multiculturalidad, y articular modelos de sociedad que superen un sistema generador de desigualdades, enfermedad y muerte. Ello implica una reflexión concienzuda sobre los derechos y las responsabilidades, tarea que compromete a toda la sociedad. 


\section{Bibliografía}

Aranda G., Patricia. 2005. Saberes relacionados con el Papanicolaou y el cáncer cervicouterino: estudio con derechohabientes del Instituto Mexicano del Seguro Social en Hermosillo, Sonora. Tesis de doctorado en Ciencias Sociales, Centro de Investigaciones y Estudios Superiores en Antropología Social.

Calvario P., José Eduardo. 2003. Masculinidad, padecimientos y accidentes por el trabajo. El caso de los jornaleros del Poblado Miguel Alemán, Costa de Hermosillo. Tesis de maestría en Ciencias Sociales, El Colegio de Sonora.

Cartwright, Elizabeth, Gilda Salazar y María del Carmen Castro. 2004. "Soy muy mujer”. Percepciones del cáncer cervicouterino en mujeres mayores: Hermosillo, Sonora y Tucson, Arizona. En Compartiendo historias de fronteras. Cuerpos, géneros, generaciones y salud, editado por Catalina Denman, Janice Monk y Norma Ojeda, 181-210. Hermosillo: El Colegio de Sonora.

Castro Luque, Ana L. 2000. Los números tienen la palabra. En Sonora 2000 a debate. Problemas y soluciones, riesgos y oportunidades, compilado por Ignacio Almada Bay, 559586. México: Cal y Arena, El Colegio de Sonora.

Castro V., Ma. del Carmen. 2006. “Aunque uno conozca sus derechos, pero si no sabe luchar...” El derecho a una atención de calidad de las mujeres con problemas mamarios desde una perspectiva sociológica. Tesis de doctorado en Ciencias Políticas y Sociales, Universidad Nacional Autónoma de México.

.1998. La práctica de la prueba del Papanicolaou y el conocimiento del cáncer cervicouterino de las mujeres de los sectores medio y popular en Hermosillo, Sonora. Estudios sociales. Revista de investigación del noroeste 8 (6): 68-84.

Castro V., Ma. del Carmen y Gilda Salazar. 2001. Elementos socioculturales en la prevención del cáncer cervicouterino: un estudio en Hermosillo, Sonora. Hermosillo: El Colegio de Sonora. de Sonora.

Cerón M., Susana Prudencia. 2005. Detection and Risk Factors for Preeclampsia in Mexico. Tesis de doctorado en Epidemiología, Universidad de Michigan.

Comisión de Salud Fronteriza México-Estados Unidos. 2003. Healthy Border (Frontera saludable) 2010: An Agenda for improving Health on the United StatesMexico Border. El Paso: Comisión de Salud Fronteriza México-Estados Unidos. http://www.borderhealth.org/files/res_63.pdf (6 de febrero de 2006). 
Consejo Nacional de Población. 2005. Sonora: Indicadores demográficos, 19902030. http://www.conapo.gob.mx/00cifras/00indicadores/26.xls (6 de febrero de 2006).

Consejo Nacional para la Prevención y Control del vIH/SIDA (CONASIDA). 2005. Panorama epidemiológico del VIH/SIDA e ITS en México. México: Secretaría de Salud.

De Keijzer, Benno. 2003. Hasta donde el cuerpo aguante: género, cuerpo y salud masculina. En La salud como derecho ciudadano. Perspectivas y propuestas desde América Latina, coordinado por C. Cáceres, M. Cueto, M. Ramos y S. Vallenas, 137-152. Lima: Universidad Peruana Cayetano Herrera.

De la Cruz, Margarita. 2004. Erradicar el hostigamiento sexual en el trabajo: una responsabilidad compartida. En Las mujeres en la maquila: trabajo, instituciones de apoyo y organización sindical, coordinado por Gabriela Grijalva Monteverde y Álex Covarrubias Valdenebro, 225-252. Hermosillo: El Colegio de Sonora, Instituto Sonorense de la Mujer.

De Souza Minayo, María Cecilia y Edinilsa Eamos de Souza. 1999. É possível prevenir a violencia? Reflexoes a partir do campo da saúde pública. Ciencia \& Saúde Coletiva 4 (1): 7-23.

Denman, Catalina A., Jill Guernsey de Zapien, Kathryn Coe, José Luis Navarro Henze, Anne Guiliano, Jennifer Stephan, Luis S. Ortega, Bogomil Djambazov, Elena Méndez Brown de Galaz, Eva Moncada y Kenneth Hatch. 1999. The Problem of Invasive Cervical Cancer along the U.S.-Mexico Border: Planning and Implementation of a Bi-national Research Project. Women \& Cancer 1 (5): 45-50.

Denman, Catalina y Armando Haro. 1990. El proceso salud/enfermedad. Revista de El Colegio de Sonora 2 (2): 261-285.

Elu, María del Carmen y Elsa Santos Pruneda. 2004. Mortalidad materna: una tragedia evitable. Perinatología y Reproducción Humana 18 (1): 44-52.

Fondo de Población de las Naciones Unidas. 2004. Estado de la población mundial 2004. El consenso de El Cairo, diez años después: población, salud reproductiva y acciones mundiales para eliminar la pobreza. Nueva York: UNFPA.

Giuliano, Anna, Catalina A. Denman, José Luis Navarro Henze, Luis Ortega y Elena Méndez Brown de Galaz. 2001. Design and Results of the USA-Mexico Border Human Papillomavirus (HPv), Cervical Dysplasia, and Chlamydia Trachomatis Study. Revista Panamericana de Salud Pública 9 (3): 172-181.

Gómez Gómez, Elsa. 2002. Equidad, género y salud: retos para la acción. Revista Panamericana de Salud Pública 11 (5/6): 454-462. 
Grown, Caren, Geeta Rao Gupta y Aslihan Kes. 2005. Taking Action: Achieving Gender Equality and Empowering Women. Achieving the Millennium Development Goals. Londres: Earthscan.

H. Congreso de la Unión, Cámara de Diputados, LIX Legislatura. 2006. Violencia feminicida en diez entidades de la República mexicana. México: Cámara de Diputados del H. Congreso de la Union-LIx Legislatura, Comisión especial para conocer y dar seguimiento a las investigaciones relacionadas con los feminicidios en la República mexicana y a la procuración de justicia vinculada.

Hernández Hernández, Dulce M., Teresa Apresa García, Isabel Alvarado Cabrera, Alejandro García Carrancá, Miriam C. Guido Jiménez, José Luis González Sánchez, Fernando Cruz Talonia, Olga A. Martínez Elizondo, Laura Ornelas Bernal y Sergio Muñoz. 2002. Virus de papiloma humano de alto riesgo (vPHAR) y neoplasia intraepitelial cervical (NIC) en mujeres de dos hospitales de la Ciudad de México. Revista de Investigación Clínica 54 (4): 299-306.

Instituto Nacional de Estadística Geografía e Informática (INEGI). 2005. Estadísticas vitales del estado de Sonora. Cuaderno no. 7. Aguascalientes: INEGI.

Instituto Nacional de Estadística Geografía e Informática e Instituto Nacional de las Mujeres. 2005. Mujeres y hombres en México, 2005. Aguascalientes: INEGI.

2004. Encuesta Nacional sobre la Dinámica de las Relaciones en los Hogares 2003 (ENDIREH). Aguascalientes: Instituto Nacional de Estadística, Geografía e Informática, Instituto Nacional de las Mujeres, Fondo de las Naciones Unidas para el Desarrollo de la Mujer.

Lamas, Marta (compiladora). 1996. Género: la construcción cultural de la diferencia sexual. México: Coordinación de Humanidades, Programa Universitario de Estudios de Género, Universidad Nacional Autónoma de México.

Magalli, Silvia. 2003. Admite gobierno desabasto parcial de anticonceptivos. Informativo CIMAC, 18 de junio. http://www.cimacnoticias.com/noticias/03jun/ 03061808.html (18 de junio de 2003).

Mayoral G., Martha Estela. 2004. El suicidio en Sonora: género y estadística, 19901997. En El amanecer del siglo y la población mexicana, coordinado por Fernando Lozano A., 753-774. Cuernavaca: Universidad Nacional Autónoma de México, Centro Regional de Investigaciones Multidisciplinarias, Sociedad Mexicana de Demografía.

Organización Mundial de la Salud. 2004. Informe mundial sobre prevención de los traumatismos causados por el tránsito: resumen. Ginebra: Organización Mundial de la Salud. 
Organización Panamericana de la Salud, Unidad de Género, Etnia y Salud. 2004. Indicadores básicos para el análisis de la equidad de género en salud. Washington: Organización Panamericana de la Salud. http://www.paho.org/ Spanish/AD/GE/indicadoresbasicos.pdf (6 de febrero de 2006).

Programa de las Naciones Unidas para el Desarrollo. 2004. Informe sobre desarrollo humano 2004. México: Mundi-Prensa. http://hdr.undp.org/ reports/global/2004/espanol/ (6 de febrero de 2006).

Secretaría de Salud. 2006. Informe Salud: México 2001-2005. Información para la rendición de cuentas. México: Secretaría de Salud.

2005. Informe Salud: México 2004. Información para rendición de cuentas. México: Secretaría de Salud.

Secretaría de Salud Pública de Sonora. 2003. Anuario estadístico de Sonora. México: Instituto Nacional de Estadística Geografía e Informática. http://www.salud sonora.gob.mx/estadisticas/ (6 de febrero de 2006).

Szasz, Ivonne. 2003. Pensando en la salud reproductiva de hombres y mujeres. En Salud reproductiva: temas y debates, editado por Mario Bronfman y Catalina Denman, 35-57. Cuernavaca: Instituto Nacional de Salud Pública.

1998. Alternativas teóricas y metodológicas para el estudio de la condición de la mujer y la salud materno-infantil. En La condición de la mujer en el espacio de la salud, compilado por Juan Guillermo Figueroa, 313-331. México: El Colegio de México.

Tirado-Gómez, Laura Leticia, Alejandro Mohar-Betancourt, Malaquías LópezCervantes, Alejandro García-Carrancá, Francisco Franco-Marina y Guilherme Borges. 2005. Factores de riesgo de cáncer cervicouterino invasor en mujeres mexicanas. Salud Pública de México 47 (5): 342-348.

Whitehead, Margaret. 1990. The Concepts and Principles of Equity and Health. Copenhagen: World Health Organization.

World Health Organization. 1997. Violence and Injury Prevention.Violence against Women: A Priority Health Issue. Geneva: World Health Organization. 